This is an author-created version of an accepted article whose final, definitive version has

been published in Lighting Research \& Technology ( http://Irt.sagepub.com/ ) by SAGE

Publications Ltd. All rights reserved. (c) The Authors.

https://doi.org/10.1177/1477153518808337

\title{
Estimating the relative contribution of streetlights, vehicles, and residential lighting to the urban night sky brightness
}

\author{
S Bará $\mathrm{PhD}^{\mathrm{a}}$, Á Rodríguez-Arós $\mathrm{PhD}^{\mathrm{b}}, \mathbf{M}$ Pérez $\mathrm{MSc}^{\mathrm{c}}, \mathbf{B}$ Tosar $\mathrm{MSc}^{\mathrm{c}}, \mathbf{R C}$ Lima $\mathrm{PhD}^{\mathrm{d}, \mathrm{e}}, \mathbf{A}$ \\ Sánchez de Miguel $\mathrm{PhD}^{\mathrm{f}}$, and J Zamorano $\mathrm{PhD}^{\mathrm{g}}$ \\ ${ }^{a}$ Facultade de Óptica e Optometría, Universidade de Santiago de Compostela, Santiago \\ de Compostela, Galicia, Spain \\ ${ }^{b}$ ETS Náutica e Máquinas, Universidade da Coruña, Galicia, Spain \\ ${ }^{c}$ Casa das Ciencias, Museos Científicos Coruñeses, A Coruña, Galicia, Spain \\ ${ }^{d}$ Escola Superior de Saúde, Politécnico do Porto, Portugal \\ ${ }^{\mathrm{e}}$ Centre for Research on Earth and Space of the University of Coimbra, Portugal \\ ${ }^{\mathrm{f}}$ Instituto de Astrofísica de Andalucía, Granada, Spain \\ ${ }^{g}$ Departamento de Física de la Tierra y Astrofísica, Facultade de Ciencias Físicas, \\ Universidad Complutense, Madrid, Spain.
}

Short title: Contributions to night sky brightness

Received 22 July 2018; Revised 31 August 2018; Accepted 1 October 2018

Under stable atmospheric conditions the brightness of the urban sky varies throughout the night following the time course of the anthropogenic emissions of light. Different types of artificial light sources (e.g. streetlights, residential, and vehicle lights) have specific time signatures, and this feature makes it possible to estimate the amount of brightness contributed by each of them. Our approach is based on transforming the time representation of the zenithal night sky brightness into a modal expansion in terms of the time signatures of the different sources of light. The modal coefficients, and hence the absolute and relative contributions of each type of source, can be estimated by means of a linear least squares fit. A practical method for determining the time signatures of different contributing sources is also described, based on wide- 
field time-lapse photometry of the urban nightscape. Our preliminary results suggest that, besides the dominant streetlight contribution, artificial light leaking out of the windows of residential buildings may account for a significant share of the timevarying part of the zenithal night sky brightness at the measurement locations, whilst the contribution of the vehicle lights seems to be significantly smaller.

Address for correspondence: Salvador Bará, Área de Óptica, Dept. Física Aplicada, Facultade de Óptica e Optometría, Universidade de Santiago de Compostela, 15782 Santiago de Compostela, Galicia, Spain.

E-mail: salva.bara@usc.es https://orcid.org/0000-0003-1274-8043

\section{Introduction}

Light pollution is a widespread phenomenon in the modern world ${ }^{1-4}$. In many places on our planet the natural levels of night darkness are being significantly disrupted, as an unwanted side-effect of the progressive extension of the use of artificial light ${ }^{5-8}$. One of the most conspicuous manifestations of this problem is the increased sky glow that hinders the observation of the starry sky and increases the radiance and irradiance levels at otherwise pristine natural sites, located many kilometres away from the urban conglomerates ${ }^{1-4,9-14}$.

Several types of artificial light sources contribute to this phenomenon. First and foremost outdoor public and private lighting systems improperly designed or installed, often producing unnecessarily high illuminances, that send a relevant fraction of light towards the sky either directly or after reflecting off the ground. Vehicle lights also contribute to the overall sky glow: a fraction of the energy emitted by the headlights propagates at angles above the horizontal, and a non-negligible amount is reflected by 
the pavement, with a combined Lambertian-directional angular pattern that depends on the pavement reflectance distribution function and on the prevailing atmospheric conditions (in particular, rain and snow). Finally, commercial and residential indoor lights, leaking out of the windows, also contribute with complex radiance patterns to the overall sky brightness.

Estimating the relative weights of these contributions is an issue of interest not only for basic science, but also for practical policy-making. A significant reduction of the present light pollution levels can only be achieved by acting on the sources, and the approaches and timing of any practicable corrective measures will necessarily be different for each of them. Whilst most efforts have been devoted to re-designing outdoor lighting systems in a more efficient way, a reappraisal of the present design of vehicle headlights and some cultural changes in the way window shutters and blinds are used in some cities could help to achieve additional reduction goals.

The estimation of the relative contribution of each type of source to the overall sky brightness can be made using two different approaches. One of them is based on carrying out a detailed census of the individual light emitters (streetlights, lighted windows of homes and shops, vehicle headlights,...) and evaluating their contributions to the night sky brightness by using suitable theoretical models and software tools to calculate the amount of light scattered back from the atmosphere in the direction to the observer ${ }^{15-23}$, taking into account the individual radiance patterns of each light source (spectral and angular), the ground and wall reflections undergone by the light before being redirected towards the sky, and the prevailing meteorological conditions.

An alternate, complementary approach that we develop in this paper is to determine directly these contributions by taking advantage of the characteristic time signature of each type of artificial source. Considered as a whole, and averaged over 
the city area, streetlights, vehicles, and industrial and domestic lights contribute to the overall sky glow with specific time-varying patterns. As we show in this work, the overall sky brightness, measured in radiometric $\left(\mathrm{Wm}^{-2} \mathrm{sr}^{-1}\right)$ or in photometric $\left(\mathrm{cd} / \mathrm{m}^{2}\right)$ units, can be rigorously expressed as a linear combination of these specific time course functions. The relative weight of each term can then be easily determined by means of a linear least-squares estimation, if the overall sky brightness is measured with sufficient time resolution and the shapes of the time signature functions are known, either by direct measurements or by indirect methods. The results from this analysis provide the absolute and relative contributions of each type of light source to the overall sky brightness at any time of the night, and can be a complementary aid for making informed decisions on public lighting policies and for modifying, if needed, the prevailing forms of artificial light use at night.

The structure of this paper is as follows. In Section 2 we briefly introduce the basis of the global time course signature approach. Section 3 describes the materials and methods we used to acquire and process the observational data gathered in the campaigns described in Section 4. A discussion of the limitations and significance of this work is carried out in Section 5, and conclusions are drawn in Section 6. Some additional formalization of the mathematical expressions used in this work can be found in the Appendix.

\section{Estimating the components of the urban night sky brightness}

In clear and moonless nights, under constant atmospheric conditions, the zenithal brightness of the sky in strongly light-polluted cities varies throughout the night 
following the time course of the emissions of artificial light. Assuming that $M$ different kinds of sources (e.g. streetlights, industrial, vehicle, ornamental, domestic lights, ... ) contribute to the overall sky glow, the time course of the zenithal night sky brightness $B(t)$, expressed in weighted radiance $\left(\mathrm{Wm}^{-2} \mathrm{sr}^{-1}\right)$ or luminance $\left(\mathrm{cd} / \mathrm{m}^{2}\right)$ units, can be written as:

$$
B(t)=\sum_{i=1}^{M} c_{i} T_{i}(t)
$$

where $T_{i}(t), i=1, \ldots, M$, are a set of functions describing the time course of the emissions of the different types of light sources, and $c_{i}$ are constants accounting for the relative contribution of each type of source to the total zenithal sky brightness recorded at the measurement site (see Appendix for formal details). The normalization and units of $c_{i}$ and $T_{i}(t)$ can be freely chosen, as long as their product in Equation (1) has the correct value in radiance or luminance units. A practical choice, among other possible options, is to normalize $T_{i}(t)$ such that it is dimensionless and equal to 1 at initial time, that is, $T_{i}(0)=1$. According to Equation (1), the relative contribution of the $k$-th type of source to the overall zenithal sky brightness at time $t, \gamma_{k}(t)$, is given by:

$$
\gamma_{k}(t)=\frac{c_{k} T_{k}(t)}{\sum_{i=1}^{M} c_{i} T_{i}(t)}
$$

From Equation (2) the relative contributions $\gamma_{k}(t)$ at any time of the night can be directly detemined, provided that the values of the constants $c_{i}$ and the time signature functions $T_{i}(t), i=1, \ldots, M$, are known. The $c_{i}$ constants, in turn, can be easily estimated (in a least-squares sense) from the measured values of the zenithal sky brightness, $B(t)$. Let us assume that the values of $B(t)$ and $T_{i}(t)$ are known for a 
sufficiently large array of time points $t_{s}, s=1, \ldots, N$, where $N$ is the total number of values of each function. Since Equation (1) applies individually for each $t_{s}$, it can be rewritten in matrix-vector form as:

$$
\mathbf{b}=\mathbf{T} \mathbf{c}
$$

where $\mathbf{b}$ is a vector of size $N \times 1$, with components $b_{s}=B\left(t_{s}\right), \mathbf{T}$ is a matrix of size $N \times M$ whose elements are given by $T_{s k}=T_{k}\left(t_{s}\right)$, and c is a $M \times 1$ vector whose components are the unknown coefficients $\left\{c_{k}\right\}$. If the number of measurements exceeds the number of unknowns, i.e. if $N>M$, the linear system in Equation (3) is overdetermined and can be solved, in the least-squares sense, by $^{24}$ :

$$
\hat{\mathbf{c}}=\mathbf{T}^{+} \mathbf{b}
$$

where $\mathbf{T}^{+}$is a suitable pseudoinverse matrix, as e.g. $\mathbf{T}^{+}=\left(\mathbf{T}^{\prime} \mathbf{T}\right)^{-1} \mathbf{T}^{\prime}$, with $\mathbf{T}^{\prime}$ standing for the transpose of $\mathbf{T}$. The symbol ${ }^{\wedge}$ indicates that $\hat{\mathbf{c}}$ is an estimate of the true vector $\mathbf{c}$ : these vectors are not expected to be strictly coincident, due to the unavoidable propagation to $\hat{\mathbf{c}}$ of the noise present in the measurements of $B(t)$ and, also, in the elements of $\mathbf{T}$, if they are determined experimentally and not from theoretical first principles. Finally, by substituting the values of the elements of $\hat{\mathbf{c}}$ for those of $\mathbf{c}$ into Equation (2) we get the estimated contribution of each type of light source to the zenithal sky brightness. If Equation (4) returns some negative coefficients, iterative linear least squares procedures with a non-negativity constraint on can be used to solve Equation (3).

\section{Materials and methods}




\subsection{Measuring the zenithal night sky brightness}

We measured the zenithal night sky brightness $B(t)$ in two field campaigns, using two different models of low-cost SQM light meters (Unihedron, Canada). These widely used devices are based on a TSL237 (TAOS, USA) high-sensitivity irradiance-tofrequency converter, with temperature correction, fitted with optics that restricts its field of view to a region of the sky with an approximately Gaussian weighting profile and a full-width at half maximum of $20^{\circ}$. The spectral band is limited to $400-650 \mathrm{~nm}$ (effective passband of the whole setup, at half the maximum sensitivity) ${ }^{25-26}$.

The readings of the SQM light meters are given in the negative logarithmic scale of magnitudes per square arcsecond (mag/arcsec $\left.{ }^{2}\right)$, a non-SI unit for the integrated spectral radiance, scaled and weighted by the spectral sensitivity of the detector ${ }^{9}$. An offset of $0.1 \mathrm{mag} / \mathrm{arcsec}^{2}$ shall be subtracted from the raw measurements, to account for the losses at the glass window of the detector housing, mostly due to Fresnel reflections at the air-glass interfaces. Due to the specific spectral sensitivity of the SQM device, the conversion between the SQM magnitudes and the corresponding mag/arcsec ${ }^{2}$ in other standard photometric bands, like, for example, the ones defined by the Johnson-Cousins $B, R$ or $V$ filters ${ }^{27}$, or the CIE photopic visual spectral efficiency function $V(\lambda)^{28}$, can only be done in an approximate way if a priori spectral information is not available ${ }^{29}$. The $\mathrm{V}$-band radiance $L_{V}\left(\right.$ in $\mathrm{Wm}^{-2} \mathrm{sr}^{-1}$ ) and the corresponding value of the $m_{V}$ magnitude in units $\mathrm{mag}_{v} / \mathrm{arcsec}^{2}$ are approximately related by ${ }^{9}$ :

$$
L_{V}\left[W \cdot m^{-2} s r-1\right]=158.1 \times 10^{\left(-0.4 m_{V}\right)} .
$$

Under the (only approximate) assumption that visible $V(\lambda)$ luminances can be directly estimated from Jonhson-Cousin $V$ radiances, the luminance $L$ in $\mathrm{cd} / \mathrm{m}^{2}$ (equivalent to $\mathrm{Ix} / \mathrm{sr}$ ) can be calculated by multiplying Equation (5) by the $683 \mathrm{Im} / \mathrm{W}$ 
luminous efficacy factor, obtaining the standard conversion formula ${ }^{4,9,21}$ :

$$
L\left[c d \cdot m^{-2}\right]=10.8 \times 10^{4} \times 10^{\left(-0.4 m_{V}\right)} .
$$

In our measurements, the zenithal night sky brightness $B(t)$ was continuously monitored throughout the night, at rates ranging from one reading every 42 seconds to one reading per minute, depending on location. These rates are considerably faster than the characteristic times in which the contributions of most types of artificial light sources, aggregated at the city level, are expected to vary; this oversampling, however, provides a high number of data points that are instrumental for attenuating the effects of the measurement noise.

\subsection{Determining the time course of the artificial light sources}

The relevant types of artificial light sources may vary from site to site, and shall be determined after analyzing the specific features of each urban area. Streetlights, vehicle headlights, and light leaking out from the windows of residential buildings are three relevant sources that shall be taken a priori into account in most cities and towns. Other sources that may contribute significant amounts of sky glow with specific time courses, for example, industrial facilities or ornamental lighting, shall also be included when relevant.

In this work we estimated the time course of the artificial components, $T_{k}(t)$, by two different methods. Detailed information about the regulation of the public outdoor lighting systems, as well as the times of the night when they are partially dimmed, where applicable, was provided by the technical services of the municipalities where this study was carried out. On the other hand, the time course of industrial, residential and vehicle lights was measured by means of wide-field time-lapse photometry of the urban nightscape, using off-the-shelf digital single-lens reflex cameras (DSLR) ${ }^{30-35}$. This technique has been successfully used by Dobler et al. ${ }^{36}$ to 
determine the aggregated behaviour of residential and commercial light sources in New York and by Meier ${ }^{37}$ to analyze the temporal profiles of urban lighting in Berlin. Here we extend this approach to the traffic flow and to some specific source types, such as outdoor industrial lighting. The time-lapse measuremens also served to check the information regarding the streetlight operation and dimming times.

The basic time-lapse procedure consists of acquiring from one or several fixed vantage points successive images of the urban nightscape, containing the basic types of sources under study (Figure 1), and extracting from these images the temporal evolution of the source radiances. 


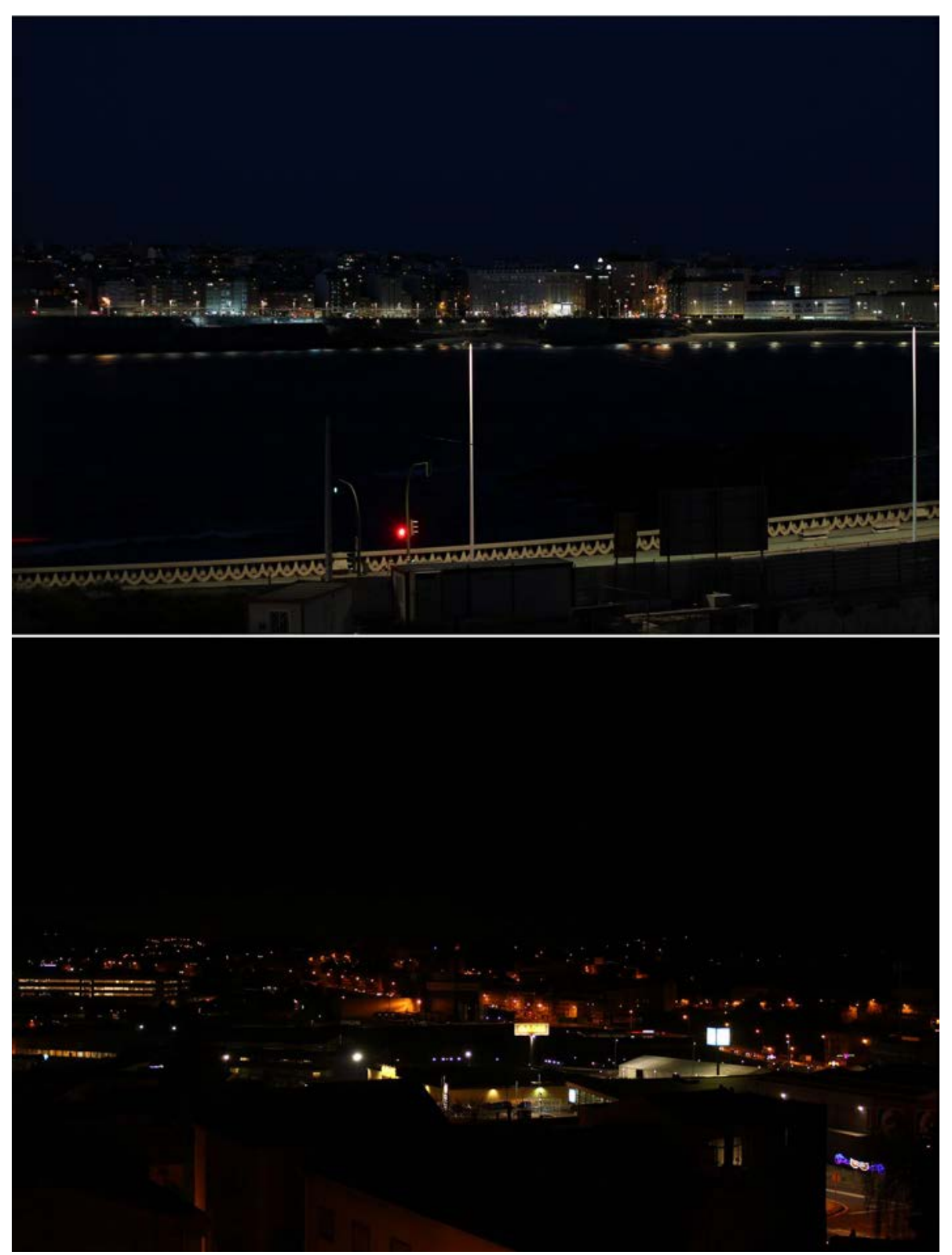

Figure 1. Examples of individual time-lapse frames taken at the two sites analyzed in our study, A Coruña (top) and Arteixo (bottom), both located in Galicia (Spain) 
The DSLR images are taken at typical rates of one frame per minute, and stored in RAW format for subsequent off-line analysis. The focal length of the camera lens is chosen to attain the desired field of view, and the ISO sensitivity, diaphragm aperture and exposure times are settled to maximize the use of the available dynamic range of the CCD or CMOS camera sensor. Details of the parameters used in our measurements can be found in Section 4.

Our time-lapse analysis workflow proceeded as follows. First, the images in the RAW proprietary camera format were converted to the open, lossless, DNG RAW format using a free application as e.g. Adobe's DNG converter ${ }^{\mathrm{TM}}$. The resulting DNG files were read with MATLAB ${ }^{\mathrm{TM}}$ (MathWorks, USA), and processed to extract the Bayer colour filter array (CFA), linearize it, apply the appropriate white balance multipliers, and interpolate each colour channel in order to get a full-resolution RGB image (demosaicing). This RGB image is then converted into graylevel luminance by means of a suitable linear combination of its RGB colour channels.

A set of binary masks was constructed, each one corresponding to the regions of the image that contain a given type of source (e.g. residential buildings windows, or vehicle traffic roads). The time-lapse luminance images are successively multiplied (pixel-wise) by each mask and the average value of the pixels corresponding to each region is calculated. The outcome is a set of linear data arrays containing the average pixel values at each time of the night and for each region. Then, the constant background light is subtracted from the arrays whose binary mask contained pixels not belonging to the sources themselves (e.g. segments of façades with no windows, or road surfaces in periods of no traffic), and all arrays are normalized such that their initial value is set to 1 . The resulting arrays are taken as an estimate of the shapes of 
$T_{k}\left(t_{s}\right)$ for $k=1, \ldots, M$ and $s=1, \ldots, N$. As a final step, and to ensure working in a homogeneous time frame when applying Equation (4), the SQM luminances (see Section 3.1) are linearly interpolated between measurements at times coincident with the time-lapse $t_{s}$ array.

\section{Results}

A proof-of-concept trial of this method was carried out over two cloudless and moonless nights in the cities of A Coruña (250.000 inh.) and Arteixo (30.000 inh.), located on the Atlantic shoreline of the North West coast of Galicia (Spain). The parameters and results of each observation night are given below. In both cases the analysis was restricted to the period comprised between the end of the evening astronomical twilight and the beginning of the morning astronomical twilight, that is, the astronomical night (Sun altitude below $-18^{\circ}$ ).

\subsection{A Coruña}

The measurements in A Coruña were carried out in the night of 27 to 28 April, 2017. The zenithal sky brightness was measured from the premises of the Casa das Ciencias Planetarium (Museos Científicos Coruñeses) located in the centre of the city, using a SQM-LU detector taking one reading every 42 seconds. The time course of the components of the artificial light emissions was determined by time-lapse photometry using the DSLR images or the urban nightscape recorded from the ETS de Náutica e Máquinas of the University of A Coruña, located $1.1 \mathrm{~km}$ to the NNW of the former location (Figure 1, top). These images were acquired at a rate of one frame per minute with a $50 \mathrm{~mm}$ fixed focal length lens set at $\mathrm{f} / 2.8$ attached to a Canon EOS 1200D Digital 
SLR standing on a tripod and set to ISO 400 and a shutter speed of $1 / 10 \mathrm{~s}$. Since the time evolution of the emissions of the outdoor public lighting system of A Coruña municipality was known in advance, only the residential and vehicle lights components were determined from these images. They were subsequently normalized as described in Section 3.2. The resulting $T_{k}(t)$ functions, as well as the SQM radiances normalized to 1 at the initial time point, are shown in Figure 2. 


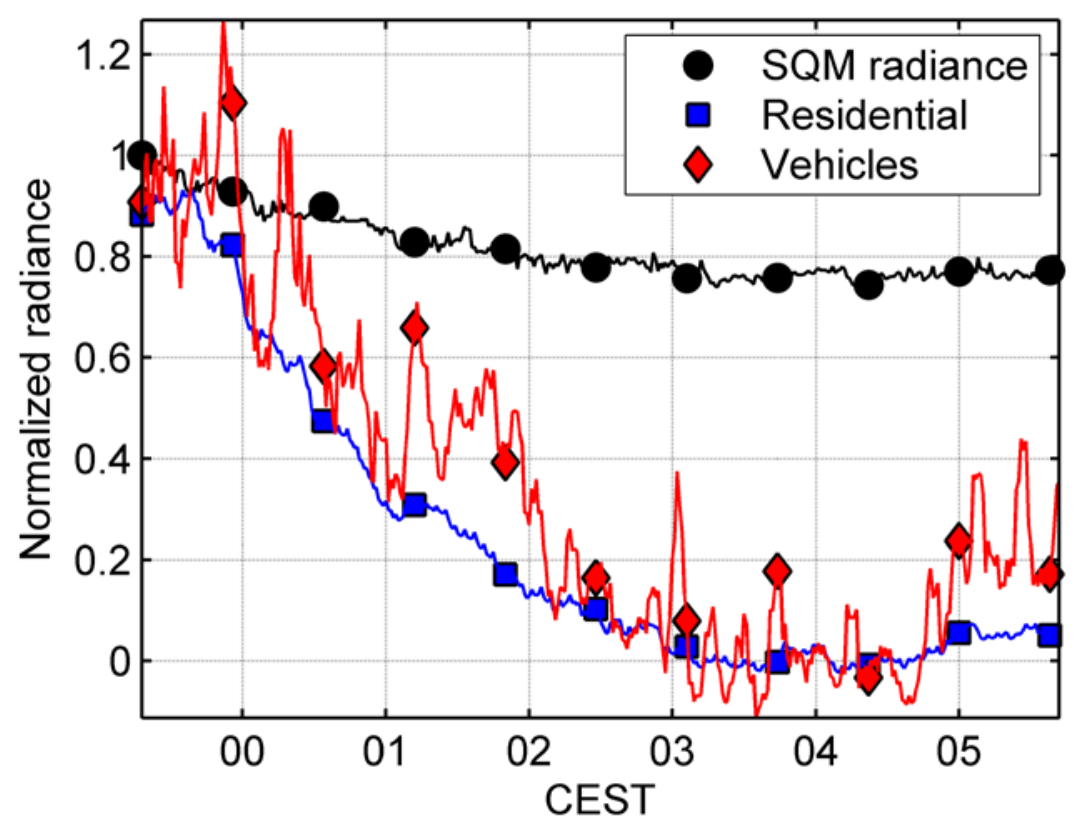

Figure 2. Normalized time course $T_{k}(t)$ of the zenithal night sky brightness (SQM radiance) and the urban emissions of light from residential buildings and vehicles in the city of A Coruña, estimated from the measurements carried out in the night of 27 to 28 April 2017. Time axis in hours, Central European Summer Time (CEST). 
The $T_{k}(t)$ functions corresponding to the residential buildings and vehicle lights shown in Figure 2 are highly fluctuating in short time scales, an artifact due to the relatively small number of individual sources of each type that can be detected within the field of view of a typical time-lapse frame. This effect is more noticeable in the case of the vehicle headlights, since the area of the images occupied by streets and roads is relative small. The overall trend of these functions, however, is deemed representative of the actual emissions taking place at the whole city level, and can be extracted from the raw signals by low-order polynomial fits, as shown in Figure 3.

The $T_{k}(t)$ functions corresponding to the public streetlight system were constructed from the information provided by the technical services of the municipality of A Coruña. Although most streetlights were kept at a constant light output from dusk to dawn, a small fraction was dimmed from 00:30 to 06:00 (CEST), and another fraction used a five-step regulation scheme: (1) from dusk to 00:00 at full light output, (2) 00:00--02:00 at reduced light output, (3) 02:00--05:00 at maximum light output reduction, (4) 05:00--06:00 at the same level as (2), and (5) from 06:00 until dawn at full light output. From this information the public streelight contribution was considered composed of four basic normalized terms: $T_{s 1}(t)=1$ for all $t$ (constant baseline component); $T_{s 2}(t)=0$ for $00: 30 \leq t<06: 00$, and 1 otherwise (dimming, in two step regulation); $T_{s 3}(t)=0$ for 00:00 $\leq t<06: 00$, and 1 otherwise (first light output reduction level of the five-step regulation); and $T_{s 3}(t)=0$ for 02:00 $\leq t<05: 00$, and 1 otherwise (second light output reduction level of the five-step regulation). 

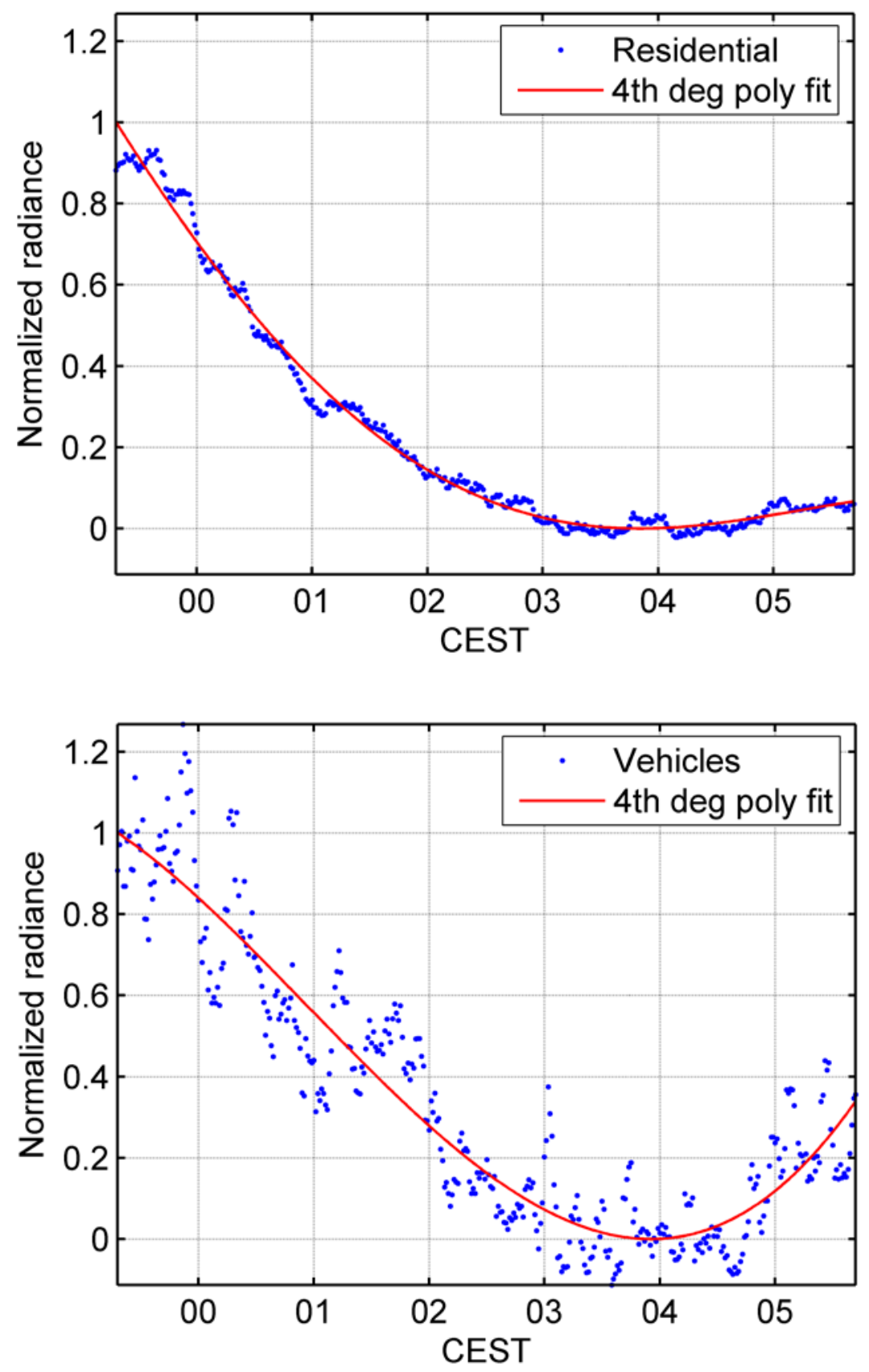

Figure 3. 4th degree polynomial fits of the residential (top) and vehicle light signals (bottom) shown in Figure 2, which were used as estimates of $T_{k}(t)$ for these types of 
sources. Time axis in hours, Central European Summer Time (CEST). 
The estimated components of the modal coefficients vector are $\hat{\mathbf{c}}=(4.41,1.22$, $0.07)$ milicandelas per square metre $\left(\mathrm{mcd} / \mathrm{m}^{2}\right)$, for constant streetlights, residential, and vehicle lights, respectively, while for the regulated (dimmed) streetlight terms the coefficients were of order 0.001 or below. Coefficients non-negativity was used as a reconstruction constraint. The estimated one-sigma uncertainties of the retrieved coefficients are $0.003,0.04$ and $0.04\left(\mathrm{mcd} / \mathrm{m}^{2}\right)$, respectively, computed from the modal noise propagators of the unconstrained linear least-squares reconstruction matrix, assuming a maximum SQM uncertainty of $0.05 \mathrm{mag} / \operatorname{arcsec}^{2}$ with constant probability distribution. The condition number of the $\mathbf{T}^{\prime} \mathbf{T}$ matrix is $3 \times 10^{3}$. From these components, and using Equations (1) and (2), the absolute and relative contribution of each type of source to the zenithal night sky brightness can be estimated for every moment of the night. Figure 4 shows the time evolution of this brightness, expressed in luminance units $\left(\mathrm{mcd} / \mathrm{m}^{2}\right)$. The reconstructed overall brightness is fairly coincident with the SQM measurements.

The relative weight of each component of the urban emissions is shown in Figure 5. The contributions of the vehicle and residential lights diminish steadily from the end of the astronomical evening twilight until about 04:00 h (CETS), increasing continuously from that moment on until the beginning of the astronomical morning twilight, although at a slower pace. The relative weight of the constant streetlight emissions follows, in consequence, the opposite trend.

The maximum residential lighting contribution takes place at the beginning of the astronomical night and amounts to a $21.5 \%\left(1.22 \mathrm{mcd} / \mathrm{m}^{2}\right)$ of the total sky brightness, whilst the vehicle lights seem to have a noticeably smaller contribution, of the order of $1.2 \%\left(0.07 \mathrm{mcd} / \mathrm{m}^{2}\right)$. At the same time the contribution of the constant streetlights $\left(4.41 \mathrm{mcd} / \mathrm{m}^{2}\right)$ reaches its minimum relative value $(77.3 \%)$. 


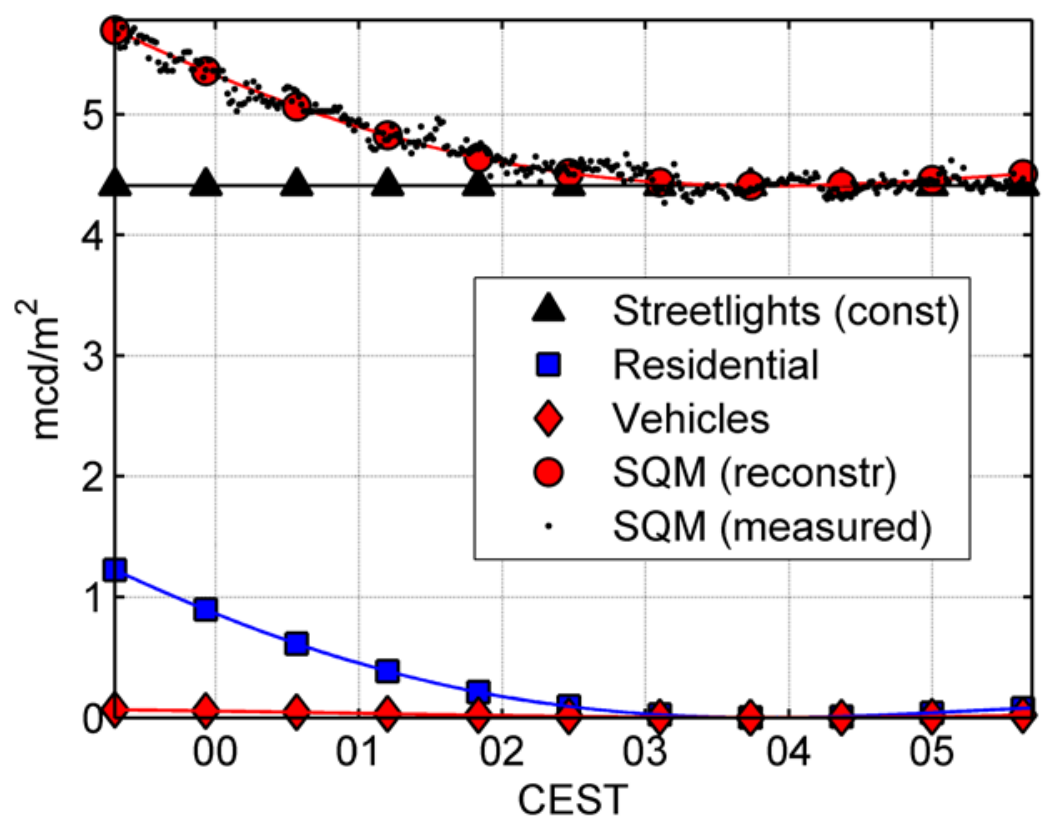

Figure 4. The measured SQM zenithal sky brightness of A Coruña, in absolute luminance units $\left(\mathrm{mcd} / \mathrm{m}^{2}\right)$, and its constant streetlight, residential and vehicle components estimated by Equation (4). SQM(reconstr) is the reconstructed zenithal luminance resulting from adding up these components as indicated in Equation (1). The regulated subset of streetlights seem to have a negligible contribution to the zenithal brightness at the measurement site and their individual curves are not displayed in this figure. 


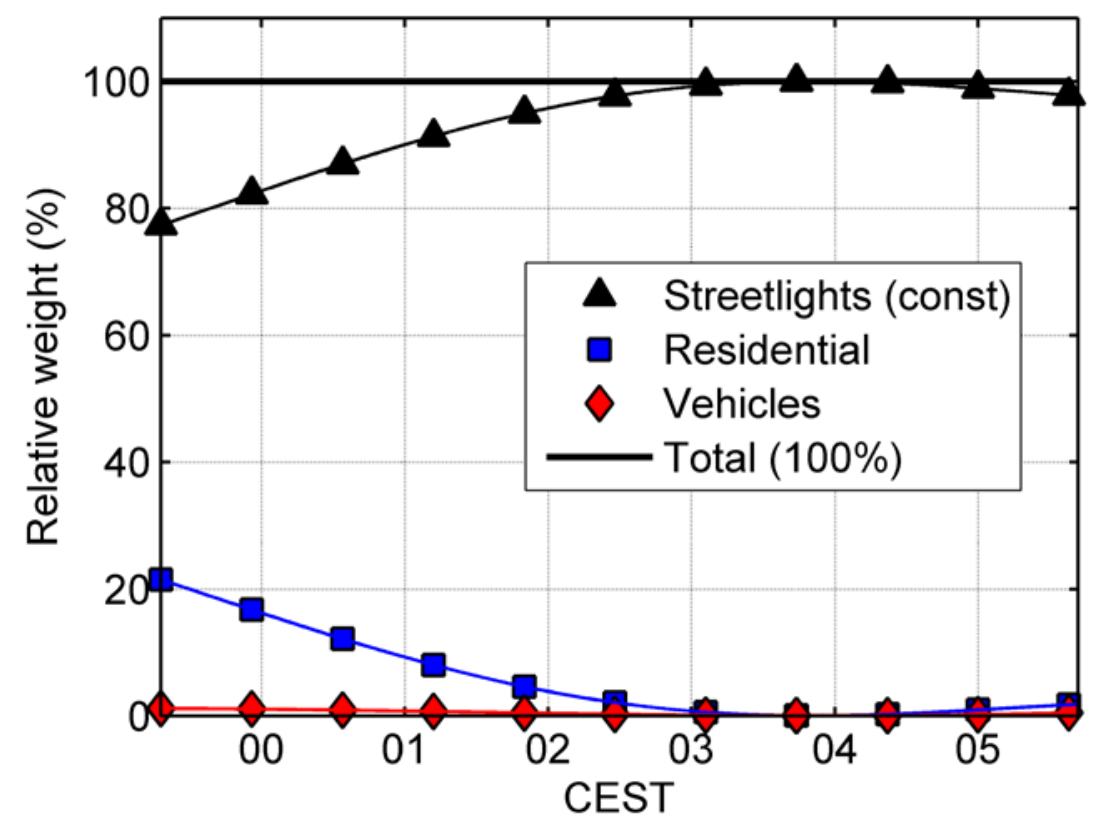

Figure 5. Relative weight of the components of the zenithal sky brightness throughout the night, based on the measurements carried out in A Coruña. The contributions of vehicle and residential lights diminish steadily from the end of the astronomical twilight after sunset until about 04:00 $\mathrm{h}$ (CEST), increasing from then on until the beginning of the astronomical twilight before sunrise. The relative weight of the constant streetlight emissions follows the opposite trend. The regulated subset of streetlights seem to have a negligible contribution to the zenithal brightness at the measurement site and their individual curves are not displayed in this figure. 


\subsection{Arteixo}

The measurements in Arteixo were made in the night of 24 to 25 February, 2017, from a vantage point located at the centre of the main urban nucleus, close to the rim of the large industrial park of Sabón (Figure 1, bottom). This site is located $10.1 \mathrm{~km}$ to the South West of the Casa das Ciencias Planetarium of A Coruña. The zenithal sky brightness measurements were made with a SQM-LU-DL detector, and the time course of the artificial light emissions was estimated from time lapse images obtained using the same camera model as in A Coruña, with an objective lens of focal length $25 \mathrm{~mm}$ stopped at $\mathrm{f} / 4$, ISO 800 and exposure time $1 / 25 \mathrm{~s}$. One frame was taken every two minutes during the whole duration of the astronomical night.

As in the previous example, Figures 6 to 9 show the raw $T_{k}(t)$ functions for residential and vehicle signals, their 4th-degree polynomial fits, the estimated absolute contribution of each source type to the overall zenithal sky brightness $\left(\mathrm{mcd} / \mathrm{m}^{2}\right)$, and the corresponding relative contributions, respectively. The time course of the strong light sources present in the industrial park, clearly distinguishable from the streetlights, vehicle and residential components, was included in the analysis as a set of four independent $T_{k}(t)$, as well as an additional one describing the nightly regulation (dimming) of a subset of streetlights (according to the information provided by the technical services of the municipality, 55\% of streetlights are dimmed at 00:00 (CET) until dawn). Industrial lighting components number 1 to 4 , as determined from the time lapse images, are progressively switched off at 21:30, 22:30 and 03:00 (CET), with an additional switch-on term at 05:30 (CET). 


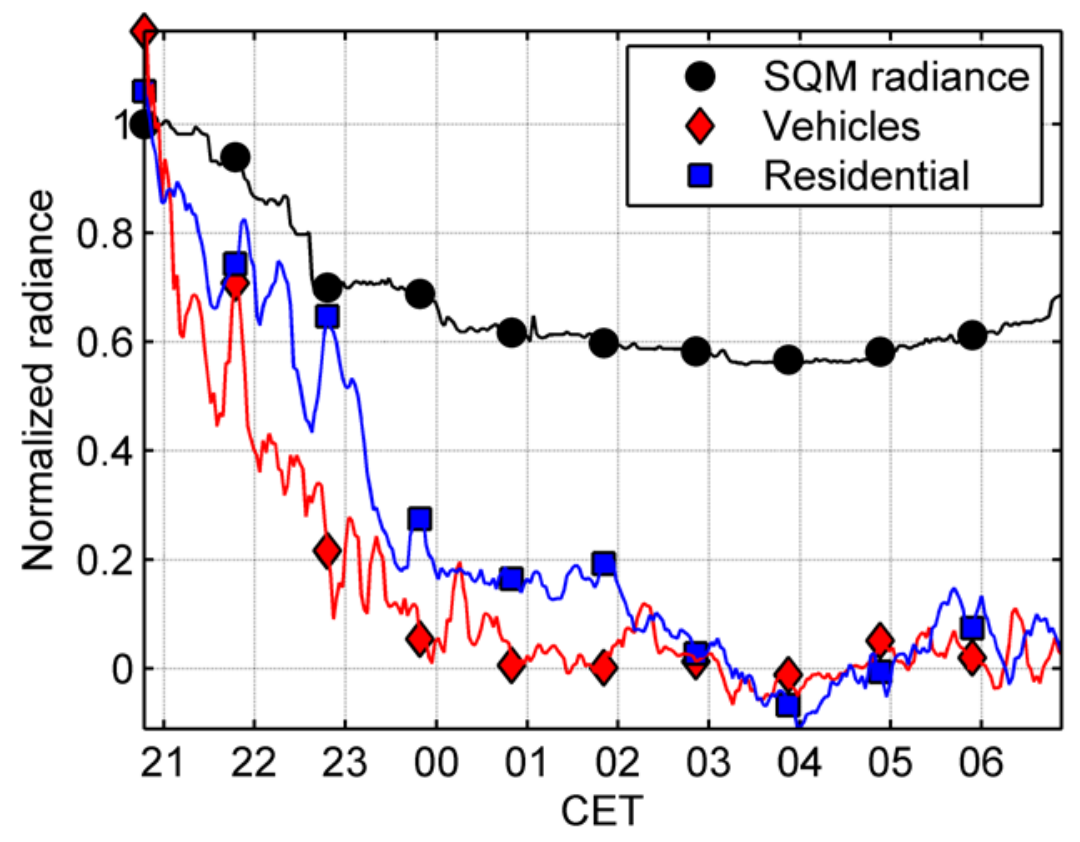

Figure 6. Normalized time courses, $T_{k}(t)$, of the zenithal night sky brightness (SQM radiance) and the urban emissions from vehicles and residential buildings in the town of Arteixo, estimated from the measurements carried out in the night of 24 to 25 February 2017. 
The estimated components of the modal coefficients vector are $\hat{\mathbf{c}}=(2.97,1.13$, $0.41,0.14,0.16,0.51,0.04,0.22) \mathrm{mcd} / \mathrm{m}^{2}$, for constant streetlights, residential, vehicle, regulated streetlights, and the four industrial light components, respectively. Nonnegativity of coefficients was used as a reconstruction constraint. The estimated onesigma uncertainties of the retrieved coefficients are 0.002, 0.03, 0.04, 0.007, 0.01, $0.008,0.004$ and $0.004\left(\mathrm{mcd} / \mathrm{m}^{2}\right)$, respectively, computed from the modal noise propagators of the unconstrained linear least-squares reconstruction matrix, assuming a maximum SQM uncertainty of $0.05 \mathrm{mag} / \operatorname{arcsec}^{2}$ with constant probability distribution. The condition number of the $\mathbf{T}^{\prime} \mathbf{T}$ matrix is $3.4 \times 10^{3}$. These components indicate that the steady contribution of the streetlights to the zenithal sky brightness is of order $2.97 \mathrm{mcd} / \mathrm{m}^{2}$ throughout the whole night, representing $55.5 \%$ of the total brightness at the beginning of the astronomical night. At the same instant of time the contributions of the remaining terms are at their maximum, being $7.7 \%\left(0.41 \mathrm{mcd} / \mathrm{m}^{2}\right)$ for vehicle headlights, $21.0 \%\left(1.13 \mathrm{mcd} / \mathrm{m}^{2}\right)$ for residential lights leaked out of the windows, $2.66 \%\left(0.14 \mathrm{mcd} / \mathrm{m}^{2}\right)$ for the regulated streetlights and $2.91 \%, 9.46 \%$ and $0.79 \%\left(0.16,0.51,0.04 \mathrm{mcd} / \mathrm{m}^{2}\right)$ for the first three industrial terms. The only exception is the fourth industrial term (switching on at 05:30 CET), whose contribution is zero at the beginning of the night. 

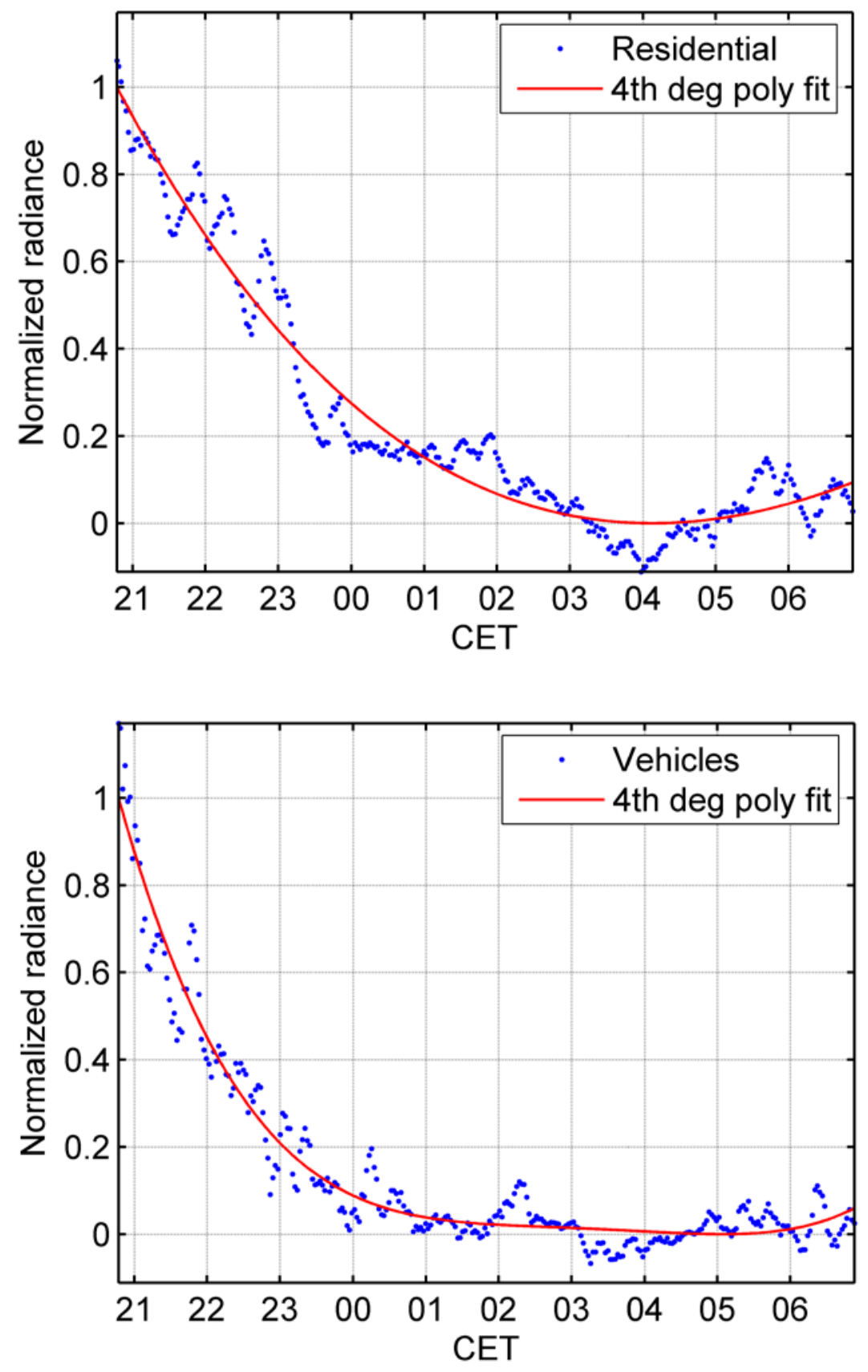

Figure 7. 4th degree polynomial fit of the residential and vehicle light signals shown in Figure 6, which were used as $T_{k}(t)$ for these sources. 


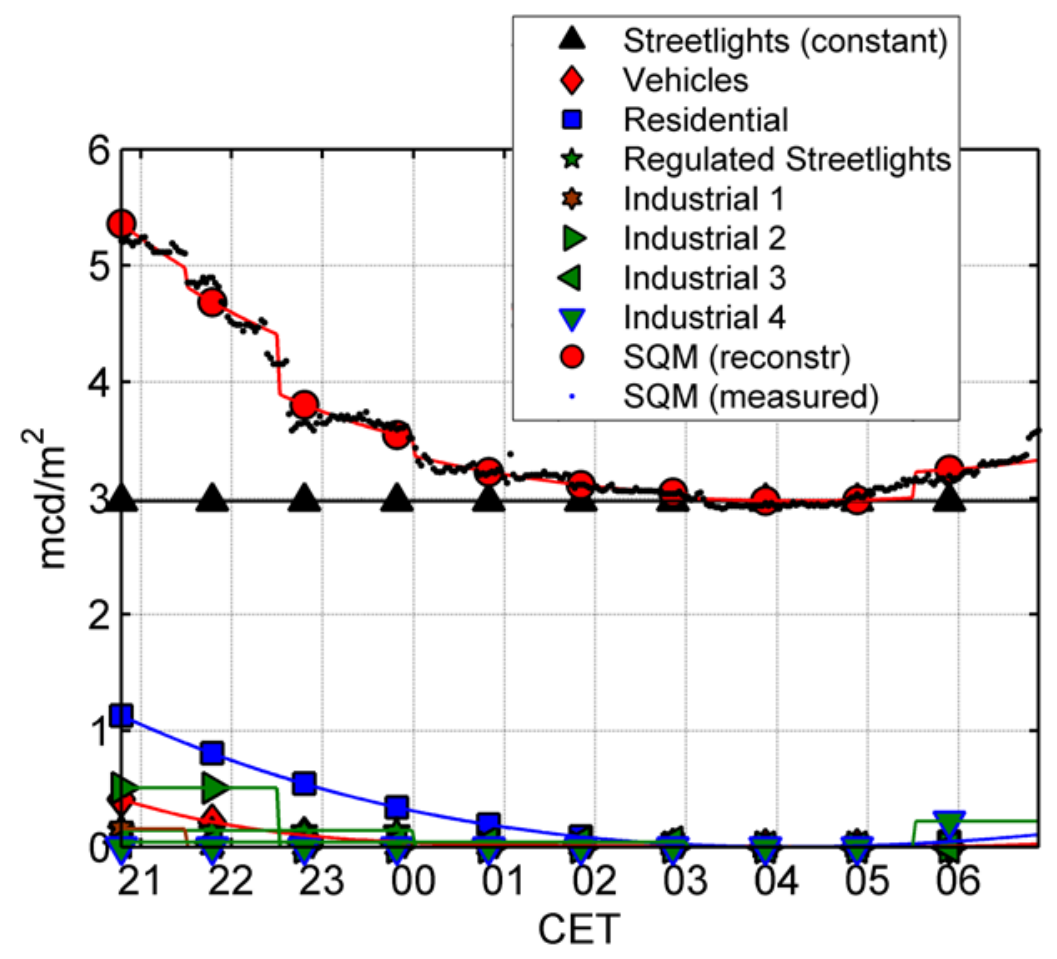

Figure 8. The measured SQM zenithal sky brightness of Arteixo, in absolute luminance units $\left(\mathrm{mcd} / \mathrm{m}^{2}\right)$, and its constant streetlights, regulated streetlights, vehicles, residential, and industrial components estimated by Equation (4). SQM(reconstr) is the reconstructed zenithal luminance obtained by adding up the estimated contribution of these components according to Equation (1). 


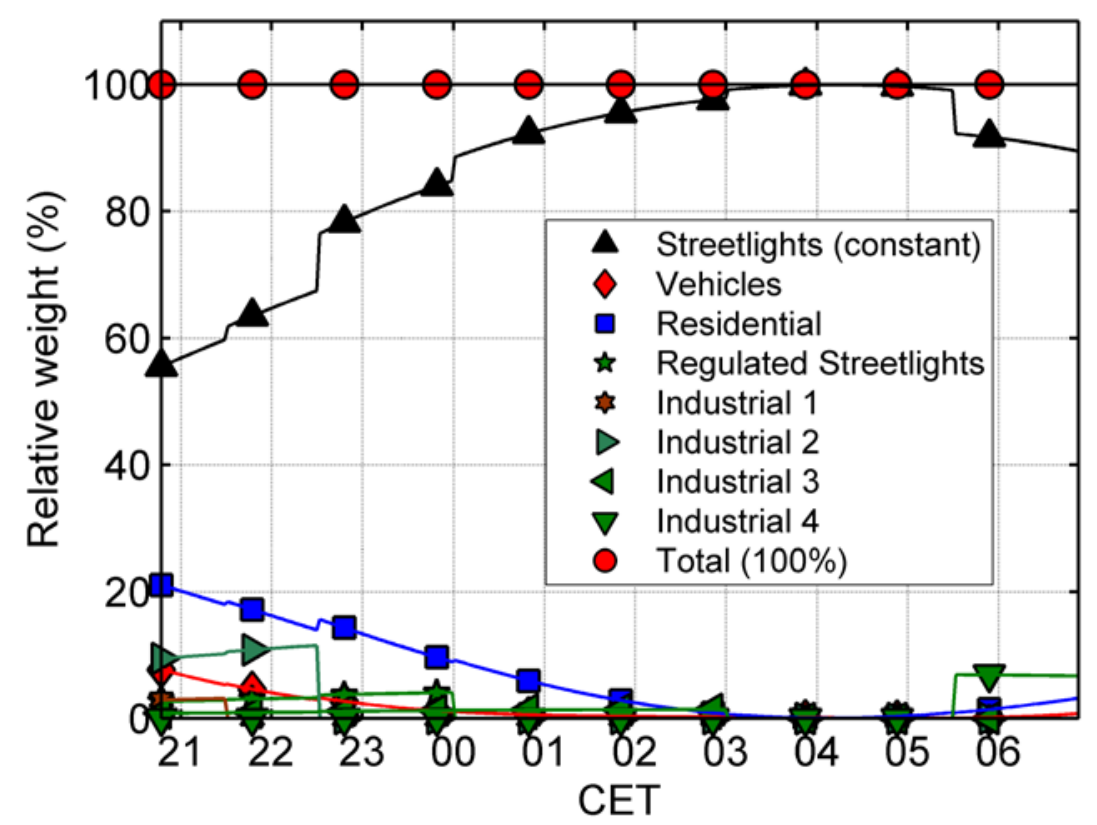

Figure 9. Relative weight of the components of the zenithal sky brightness troughout the night, based on the measurements carried out in Arteixo. As it was observed in A Coruña, the contributions of vehicle and residential lights diminish steadily from the end of the astronomical twilight after sunset until about 04:00 h (CEST), increasing from then on until the beginning of the astronomical morning twilight. The relative weight of the constant streetlight emissions follows the opposite trend. 


\section{Discussion}

It has been previously reported that the zenithal night sky brightness in urban areas tends to decrease in the first half of the night, due to the progressive switch-off of residential and industrial lights, and the reduction of the vehicle traffic flows. Typical darkening rates reported in studies carried out in different regions of the world are of order $4.5 \%$ per hour ${ }^{2}$, that is, about $0.05 \mathrm{mag} /\left(\operatorname{arcsec}^{2} \cdot h\right)$. According to these results, the absolute difference between the brightest and the darkest point in the night may easily reach $0.3 \mathrm{mag} / \mathrm{arcsec}^{2}$, amounting to a reduction of $\sim 25 \%$ in the zenithal brightness of the sky. In the examples presented in Section 4, the reduction of luminance was $26 \%$ for A Coruña and $45 \%$ for the industrial site of Arteixo. Previous studies on the zenithal sky brightness of Madrid (Spain) reported a reduction of order $49 \%$, for an estimated composition of the emissions to the upper hemisphere of $54 \%$ streetlights, $36 \%$ ornamental and commercial lights and about $9 \%$ residential lights ${ }^{13}$. This variability may be due to the different estimation methods, and may also reflect the specific light source composition and time dynamics of different cities and towns.

All observations suggest, however, that the variable components are a relevant fraction of the overall night sky brightness. Acting on this variable part of the anthropogenic emissions of light may help to attain significant improvements of the current light pollution levels, complementing the necessary reduction of the upward light emissions produced by streetlights, which still represent the biggest contribution to the artificial night sky brightness. A key issue for planning and implementing any set of remediation measures is to identify the relative contribution of each type of source. Our preliminary results suggest that the light leaked out of residential building 
windows may have a relevant share in the total amount of urban emissions, especially in the first hours of the night. The outwards emissions from an individual window can be quantitatively estimated using suitable multiple-reflection models of indoor lighting ${ }^{38-39}$. A judicious use of blinds and curtains at times when looking outdoors is not required nor desired may significantly alleviate the overall light emissions from residential buildings. In contrast, the contribution of vehicle lights to the overall sky brightness seems to be significantly smaller, at least in the measurement locations used in our study. Direct illumination from vehicle lights, however, has been recently identified as a growing source of ecological impacts ${ }^{40}$.

The results presented in Section 4 should be taken as an illustration of the application of the proposed method, rather than as a final and sharply-cut statement on the relative contribution of the different types of artificial sources to the urban sky brightness. As an important remark, note that these contributions are expected to vary from point to point as the observer moves across the city and beyond: the night sky brightness (and its composition in terms of the contributions of elementary source types) varies across the territory due to the change in the relative positions of the sources and the observer, and to the particular form of the angular emission function of the sources. The relative contribution of each source is also expected to depend on the particular direction of the sky in which the measurements are made.

Both the method itself and the particular examples reported here suffer from some limitations that deserve further consideration. The core of the method is the reduction of the dimensionality of the description of the zenithal sky brightness, achieved by passing from a time-based representation $B(t)$ to a modal coefficientsbased one, $c_{i}$, in terms of the time signatures of each contributing type of source $T_{i}(t)$, and the subsequent estimation of the modal coefficients from a least-squares fit 
to a sufficiently large discrete set of measurements made at times $t_{s}, s=1, \ldots, N$. The main problem associated with this approach, from a basic standpoint, is the lack of orthogonality of the time signature functions, in the sense that $\sum_{s=1}^{N} T_{i}\left(t_{s}\right) T_{k}\left(t_{s}\right) \neq K_{i} \delta_{i k}$, being $K_{i}$ a real positive number and $\delta_{i k}$ the Kronecker-delta function. This lack of orthogonality forces the solutions of Equation (4) to be highly dependent on the number and type of terms included in the sky brightness description Equation (1). An accurate estimation of the relative brightness contributions is therefore contingent upon the correct identification of all relevant types of contributing sources. Note that in this work we have included the main types of sources known to be relevant in each location, as deduced from the processed content of the time lapse images and the information provided by the technical services of the corresponding municipalities. Other locations may require including additional terms, as e.g. light sources mounted externally on residential buildings that follow specific time schedules across the night. Besides, this lack of orthogonality, especially noticeable for the residential and vehicle lighting contributions due to the similarity of their time courses, puts stronger demands on the numerical precision required for an accurate solution of Equation (4), and may help to increase the noise propagation.

From an experimental viewpoint, the main requirement associated with this approach is the need of finding a suitable vantage point to determine with sufficient accuracy and precision the time course of the relevant artificial sources. This is particularly true of the vehicle lights signal, since the overall field of view corresponding to the streets and roads in the urban nightscape imagery is generally smaller than the one corresponding to windows, and the traffic flow at the street scale (not so at the metropolitan area scale) is highly fluctuating in time. In our present 
study the time-lapse images were taken from a single location, trying to encompass all kinds of relevant light sources: a straightforward improvement would be to acquire simultaneous time-lapse images from a variety of urban locations, each one containing a sufficiently large sample of individual sources of a given kind. Since the zenithal sky brightness responds to the overall emissions of the urban area, the key issue is finding locations sufficiently representative of the average behaviour of the city at large.

In this work we did not address the spectral composition of the light scattered back from urban skies to the observer. The spectral composition of the light emitted by urban sources can be determined by using hyperspectral imagery, either ground-based or airborne (see Dobler et al. ${ }^{41}$ and Alamús et $a l .{ }^{42}$, and references contained therein).

Putting aside these limitations, the results presented in this paper show the feasibility of the proposed approach, and suggest the relevance of residential lights in the build up of the overall levels of artificial skyglow in urban areas. Further observational campaigns are necessary to confirm or otherwise contradict this finding.

\section{Conclusions}

The zenithal night sky brightness in urban areas varies throughout the night following the changing time course of the anthropogenic emissions of light. Different types of artificial light sources (e.g. streetlights, residential, and vehicle lights) show specific time signatures, and this feature allows for an estimation of the amount of sky brightness contributed by each of them. The method described in this paper consists of reducing drastically the dimensionality of the problem by transforming the timebased representation of the zenithal sky brightness into a modal coefficients one, in 
terms of the time course signature of each source type. The modal coefficients, and hence the absolute and relative contributions of each light source, can be estimated by linear least-squares fits of the modal expansion to the measured values of the zenithal sky brightness. The zenithal sky brightness may be monitored using conventional lowcost light meters. The time course of the sources can be determined from wide-field time-lapse photometry. The preliminary results shown in this paper suggest that artificial light leaking out of the windows of residential buildings accounts for a significant share of the varying part of the zenithal sky brightness, whilst the contribution of vehicle lights is significantly smaller. Further studies are required to confirm this point.

\section{Acknowledgments}

This work was funded by grant ED431B 2017/64, Xunta de Galicia/FEDER (S.B.) and partly developed within the framework of the Spanish Network for Light Pollution Studies, REECL (AYA2015-71542-REDT). Thanks are given to Casa das Ciencias Planetarium of A Coruña for the SQM data of the night of 27 to 28 April, 2017, and to Mrs V. Rivas for the kind assignment of her office at the ETS Naútica y Máquinas as a vantage point for recording the time-lapses in A Coruña. Special thanks are due to the lighting technical departments of A Coruña and Arteixo municipalities for providing detailed information about the dimming schedules of their streetlight systems. J.Z. acknowledges funding by the Spanish MICINN AYA2016-75808-R, by the Madrid Regional Government through the SpaceTec Project (S2013/ICE-2822), and by STARS4ALL, a project funded by the European Union H2020-ICT-2015-688135. R.L. acknowledges CITEUC is funded by National Funds through FCT - Foundation for 
Science and Technology (project: UID/Multi/00611/2013) and FEDER - European Regional Development Fund through COMPETE 2020 Operational Programme Competitiveness and Internationalization (project: POCI-01-0145-FEDER-006922). 


\section{References}

1. Cinzano P, Falchi F, Elvidge C. The first world atlas of the artificial night sky brightness. Monthly Notices of the Royal Astronomical Society 2001; 328: 689707.

2. Falchi F, Cinzano P, Duriscoe D, Kyba CCM, Elvidge CD, Baugh K, Portnov BA, Rybnikova NA, Furgoni R. The new world atlas of artificial night sky brightness. Science Advances 2016; 2: e1600377.

3. Falchi F, Cinzano P, Duriscoe D, Kyba CCM, Elvidge CD, Baugh K, Portnov B, Rybnikova NA, Furgoni R. Supplement to: The New World Atlas of Artificial Night Sky Brightness. GFZ Data Services 2016. DOI:10.5880/GFZ.1.4.2016.001

4. Kyba CCM et al. Worldwide variations in artificial skyglow. Scientific Reports 2015; 5: 8409.

5. Davies TW, Duffy JP, Bennie J, Gaston KJ. The nature, extent, and ecological implications of marine light pollution. Frontiers in Ecology and the Environment 2014; 12(6): 347-355.

6. Davies TW, Duffy JP, Bennie J, Gaston KJ. Stemming the tide of light pollution encroaching into marine protected areas. Conservation Letters 2016; 9(3) :164171.

7. Hölker F, Wolter C, Perkin EK, Tockner K. Light pollution as a biodiversity threat. Trends in Ecology and Evolution 2010; 25: 681-682.

8. Hölker F et al. The dark side of light: a transdisciplinary research agenda for light pollution policy. Ecology and Society 2010; 1(4): 13.

9. Bará S. Anthropogenic disruption of the night sky darkness in urban and rural areas. 
Royal Society Open Science 2016; 3: 160541.

10. Biggs JD, Fouché T, Bilki F, Zadnik MG. Measuring and mapping the night sky brightness of Perth, Western Australia. Monthly Notices of the Royal Astronomical Society 2012; 421: 1450-1464.

11. Espey B, McCauley J. Initial Irish light pollution measurements and a new sky quality meter-based data logger. Lighting Research and Technology 2014; 46(1): 67-77.

12. Pun CSJ, So CW. Night-sky brightness monitoring in Hong Kong. Environmental Monitoring and Assessment 2012; 184(4): 2537-2557.

13. Sánchez de Miguel A. Variación espacial, temporal y espectral de la contaminación lumínica y sus fuentes: Metodología y resultados. PhD dissertation, Universidad Complutense de Madrid (Spain), 2015. doi: 10.13140/RG.2.1.2233.7127

14. Zamorano J, Sánchez de Miguel A, Ocaña F, Pila-Díez B, Gómez Castaño J, Pascual S, Tapia C, Gallego J, Fernández A, Nievas M. Testing sky brightness models against radial dependency: A dense two dimensional survey around the city of Madrid, Spain. Journal of Quantitative Spectroscopy and Radiative Transfer 2016; 181: 5266.

15. Aubé M. Physical behaviour of anthropogenic light propagation into the nocturnal environment. Philosophical Transactions of the Royal Society B 2015; 370: 20140117.

16. Aubé $M$, Kocifaj $M$. Using two light-pollution models to investigate artificial sky radiances at Canary Islands observatories. Monthly Notices of the Royal Astronomical Society 2012; 422: 819-830.

17. Cinzano $P$, Elvidge $C D$. Night sky brightness at sites from DMSP-OLS satellite measurements. Monthly Notices of the Royal Astronomical Society 2004; 353 : 
1107-1116.

18. Cinzano P, Falchi F. The propagation of light pollution in the atmosphere. Monthly Notices of the Royal Astronomical Society 2012; 427: 3337-3357.

19. García M. Metodologia ramal-UPC para analisis cuantitativo de la contaminacion luminica. In XXXV Simposium del Comité Español de Iluminación CEI, Pontevedra (Galicia, Spain) 2009.

20. García M, Conzález N. Influencia de la luz privada doméstica sobre la fuente de la contaminación lumínica. Architecture, City and Environment 2016; 11(31): 93-120.

21. Garstang RH. Model for artificial night-sky illumination. Publications of the Astronomical Society of the Pacific 1986; 98: 364-375.

22. Kocifaj M. Light-pollution model for cloudy and cloudless night skies with groundbased light sources. Applied Optics 2007; 46: 3013-3022.

23. Kocifaj M. A review of the theoretical and numerical approaches to modeling skyglow: Iterative approach to RTE, MSOS, and two-stream approximation. Journal of Quantitative Spectroscopy and Radiative Transfer 2016; 181: 2-10.

24. Liebelt PB. An Introduction to Optimal Estimation. Reading, MA: Addison-Wesley: 1967: pp. 148-149.

25. Cinzano P. Night Sky Photometry with Sky Quality Meter. Internal Report No.9, v.1.4. Istituto di Scienza e Tecnologia dell'Inquinamento Luminoso (ISTIL), 2005.

26. Pravettoni $M$, Strepparava D, Cereghetti N, Klett S, Andretta M, Steiger M. Indoor calibration of Sky Quality Meters: Linearity, spectral responsivity and uncertainty analysis. Journal of Quantitative Spectroscopy and Radiative Transfer 2016; 181 : 74-86.

27. Bessell MS. UBVRI photometry II: The Cousins VRI system, its temperature and absolute flux calibration, and relevance for two-dimensional photometry. 
Publications of the Astronomical Society of the Pacific 1979; 91: 589-607.

28. Commision Internationale de l'Éclairage. CIE $19882^{\circ}$ Spectral Luminous Efficiency Function for Photopic Vision. Vienna: CIE: 1990.

29. Sánchez de Miguel A, Aubé M, Zamorano J, Kocifaj M, Roby J, Tapia C. Sky Quality Meter measurements in a colour-changing world. Monthly Notices of the Royal Astronomical Society 2017; 467(3): 2966-2979.

30. Aceituno J, Sánchez SF, Aceituno FJ, Galadí-Enríquez D, Negro JJ, Soriguer RC, Sánchez-Gómez G. An all-sky transmission monitor: ASTMON. Publications of the Astronomical Society of the Pacific 2001; 123: 1076-1086.

31. Duriscoe DM, Luginbuhl CB, Moore CA. Measuring night-sky brightness with widefield CCD camera. Publications of the Astronomical Society of the Pacific 2007; 119: 192-213.

32. Jechow A, Kolláth Z, Lerner A, Hänel A, Shashar N, Hölker F, Kyba CCM. Measuring light pollution with fisheye lens imagery from a moving boat - a proof of concept. International Journal of Sustainable Lighting 2017; 19: 15-25.

33. Jechow A, Ribas SJ, Canal-Domingo R, Hölker F, Kolláth Z, Kyba CCM. Tracking the dynamics of skyglow with differential photometry using a digital camera with fisheye lens. Journal of Quantitative Spectroscopy and Radiative Transfer 2018; 209: 212-223.

34. Kolláth Z. Measuring and modelling light pollution at the Zselic Starry Sky Park. Journal of Physics: Conference Series 2010; 218: 012001.

35. Rabaza O, Galadí-Enríquez D, Espín-Estrella A, Aznar-Dols F. All-Sky brightness monitoring of light pollution with astronomical methods. Journal of Environmental Management 2010; 91: 1278e1287.

36. Dobler G, Ghandehari M, Koonin SE, Nazari R, Patrinos A, Sharma MS, Tafvizi A, Vo 
HT, Wurtele JS. Dynamics of the urban nightscape. Information Systems 2015; 54: $115-126$.

37. Meier J. Temporal profiles of urban lighting: Proposal for a research design and first results from three sites in Berlin. International Journal of Sustainable Lighting 2018; 20(1): 11- 28.

38. Bará S, Escofet J. On lamps, walls, and eyes: the spectral radiance field and the evaluation of light pollution indoors. Journal of Quantitative Spectroscopy and Radiative Transfer 2018; 205: 267-277.

39. Darula S. Windows in buildings: potential source of light pollution. In Proceedings of the International Conference on Light Pollution Theory, Modelling and Measurements, 15-18 April, 2013, Smolenice, Slovak Republic, 2013. pp. 51-56.

40. Gaston KJ, Holt LA. Nature, extent and ecological implications of night-time light from road vehicles. Journal of Applied Ecology 2018; 55:2296-2307.

41. Dobler G, Ghandehari M, Koonin SE, Sharma MS. A hyperspectral survey of New York City lighting technology. Sensors 2016; 16: 2047.

42. Alamús R, Bará S, Corbera J, Escofet J, Palà V, Pipia L, Tardà A. Ground-based hyperspectral analysis of the urban nightscape. ISPRS Journal of Photogrammetry and Remote Sensing 2017; 124: 16-26. 


\section{APPENDIX}

Some formal details for arriving at Equation (1) are described here. Let $L(\lambda ; \boldsymbol{\alpha}, t)$ be the spectral radiance of the sky at time $t$ in the direction $\alpha$ (a two-dimensional vector whose components are e.g. the altitude and azimuth of the points of the sky measured in the observer reference frame) and wavelength $\lambda$, expressed in units $\mathrm{Wm}^{-2} \mathrm{sr}^{-1} \mathrm{~nm}^{-1}$. The artificial component of this spectral radiance is the sum of the sky radiances produced by all individual artificial light sources present in the region surrounding the observer. If $M$ different kinds of light sources $L_{i}(\lambda ; \boldsymbol{\alpha}, t), i=1, \ldots, M$, can be identified (e.g. streetlights, residential, vehicle lights,...) each one being characterized by a particular aggregate spectral composition and a specific time course, the overall radiance can be written as:

$$
L(\lambda ; \boldsymbol{\alpha}, t)=\sum_{i=1}^{M} L_{i}(\lambda ; \boldsymbol{\alpha}, t) .
$$

If the atmospheric conditions remain stable throughout the night (or, as a less stringent condition, if the changing atmospheric conditions affect in the same way to the fraction of light scattered towards the observer for all types of light sources), and if the aggregated angular emission pattern of each type of source does not vary significantly throughout the night (a reasonable assumption, due to the high number of individual sources with random orientations that build up the emission of each source class), the sky radiances $L_{i}(\lambda ; \boldsymbol{\alpha}, t)$ will be proportional to the aggregated emitted spectral density flux, $\Phi_{i}(\lambda ; t)\left(\mathrm{W} \cdot \mathrm{nm}^{-1}\right)$, at each instant $t$. Under these assumptions, the coefficient of proportionality, $g_{i}(\lambda, \boldsymbol{\alpha})$, that depends on the kind of source $(i)$, the wavelength $(\lambda)$ and the direction in the sky $(\boldsymbol{\alpha})$, will not depend on 
time. We have, then:

$$
L_{i}(\lambda ; \boldsymbol{\alpha}, t)=g_{i}(\lambda, \boldsymbol{\alpha}) \Phi_{i}(\lambda ; t) .
$$

On the other hand the measured brightness, $B(t)$, is the result of integrating the radiance $L(\lambda ; \boldsymbol{\alpha}, t)$ over the field of view of the detector, characterized by the weighting function $F(\boldsymbol{\alpha})$, and subsequently integrating the result over wavelengths, weighted by the spectral sensitivity of the detector, $V(\lambda)$. The (noiseless) brightness measurements can hence be described by:

$$
B(t)=\int_{\Omega 0}^{\infty} \int_{0}^{\infty} L(\lambda ; \boldsymbol{\alpha}, t) F(\boldsymbol{\alpha}) V(\lambda) \mathrm{d} \lambda \mathrm{d}^{2} \boldsymbol{\alpha}=\sum_{i=1}^{M} \int_{\Omega 0}^{\infty} \int_{0}^{\infty} L_{i}(\lambda ; \boldsymbol{\alpha}, t) F(\boldsymbol{\alpha}) V(\lambda) \mathrm{d} \lambda \mathrm{d}^{2} \boldsymbol{\alpha},
$$

where $\mathrm{d}^{2} \boldsymbol{\alpha}=\cos (h) \mathrm{d} h \mathrm{~d} \phi$, with $h$ standing for altitude and $\phi$ for azimuth, is the solid angle element. The solid angle integral is formally carried out over the celestial hemisphere $\Omega$, spanning $2 \pi$ sr. Substituting Equation (A2) into Equation (A3) we get:

$$
B(t)=\sum_{i=1}^{M} \int_{\Omega 0}^{\infty} g_{i}(\lambda, \boldsymbol{\alpha}) \Phi_{i}(\lambda ; t) F(\boldsymbol{\alpha}) V(\lambda) \mathrm{d} \lambda \mathrm{d}^{2} \boldsymbol{\alpha},
$$

and, after integrating over the angular variables:

$$
B(t)=\sum_{i=1}^{M} \int_{0}^{\infty} G_{i}(\lambda) V(\lambda) \Phi_{i}(\lambda ; t) \mathrm{d} \lambda
$$

where $G_{i}(\lambda)=\int_{\Omega} g_{i}(\lambda, \boldsymbol{\alpha}) F(\boldsymbol{\alpha}) \mathrm{d}^{2} \boldsymbol{\alpha}$ is the weighted integral of $g_{i}(\lambda, \boldsymbol{\alpha})$ over the $2 \pi \mathrm{sr}$ celestial hemisphere above the observer.

Finally, if the spectral composition of the radiance emitted by each type of source does not vary throughout the night (note that the different types of sources are defined, among other criteria, by this condition), the aggregated spectral radiant flux can be factored out as: 


$$
\Phi_{i}(\lambda ; t)=\tilde{\Phi}_{i}(\lambda) T_{i}(t)
$$

where $T_{i}(t)$ is the function describing the time course of the emissions and $\widetilde{\Phi}_{i}(\lambda)$ is the spectral composition of the $i$-th type of source, which, by assumption, does not depend on time. The scaling and dimensions of $T_{i}(t)$ and $\tilde{\Phi}_{i}(\lambda)$ can be assigned arbitrarily, provided that their product gives the correct value and units for $\Phi_{i}(\lambda ; t)$. A useful choice is to identify $\widetilde{\Phi}_{i}(\lambda)$ with the overall spectral flux $\left(W \cdot n m^{-1}\right)$ emitted at a given time $t_{0}$ of the night, $\widetilde{\Phi}_{i}(\lambda) \equiv \Phi_{i}\left(\lambda ; t_{0}\right)$, in which case the time course signature $T_{i}(t)$ turns out to be dimensionless and normalized such that $T_{i}\left(t_{0}\right)=1$.

From Equation (A5) and Equation (A6) we can write:

$$
B(t)=\sum_{i=1}^{M}\left[\int_{0}^{\infty} C_{i}(\lambda) V(\lambda) \tilde{\Phi}_{i}(\lambda) \mathrm{d} \lambda\right] T_{i}(t),
$$

and hence we get Equation (1)

$$
B(t)=\sum_{i=1}^{M} c_{i} T_{i}(t)
$$

where

$$
c_{i}=\int_{0}^{\infty} C_{i}(\lambda) V(\lambda) \widetilde{\Phi}_{i}(\lambda) \mathrm{d} \lambda
$$

are constant coefficients that do not depend on time, and whose units are the same as the units of $B(t)$. According to the recommended SI practice, $B(t)$ is usually expressed as a weighted radiance $\left(\mathrm{Wm}^{-2} \mathrm{sr}^{-1}\right)$, specifying the photometric band $V(\lambda)$ used for the measurements. Note that when this band is coincident with the photopic (or scotopic) $\mathrm{CIE}$ spectral efficacy function of the human visual system, the brightness can be equivalently described in the SI luminous units $\mathrm{cd} / \mathrm{m}^{2}$. 


\section{Figure captions}

Figure 1. Examples of individual time-lapse frames taken at the two sites analyzed in our study, A Coruña (top) and Arteixo (bottom), both located in Galicia (Spain)

Figure 2. Normalized time course $T_{k}(t)$ of the zenithal night sky brightness (SQM radiance) and the urban emissions of light from residential buildings and vehicles in the city of A Coruña, estimated from the measurements carried out in the night of 27 to 28 April 2017. Time axis in hours, Central European Summer Time (CEST).

Figure 3. 4th degree polynomial fits of the residential (top) and vehicle light signals (bottom) shown in Figure 2, which were used as estimates of $T_{k}(t)$ for these types of sources. Time axis in hours, Central European Summer Time (CEST).

Figure 4. The measured SQM zenithal sky brightness of A Coruña, in absolute luminance units $\left(\mathrm{mcd} / \mathrm{m}^{2}\right)$, and its constant streetlight, residential and vehicle components estimated by Equation (4). SQM(reconstr) is the reconstructed zenithal luminance resulting from adding up these components as indicated in Equation (1). The regulated subset of streetlights seem to have a negligible contribution to the zenithal brightness at the measurement site and their individual curves are not displayed in this figure.

Figure 5. Relative weight of the components of the zenithal sky brightness throughout the night, based on the measurements carried out in A Coruña. The contributions of vehicle and residential lights diminish steadily from the end of the astronomical twilight after sunset until about 04:00 $\mathrm{h}$ (CEST), increasing from then on until the beginning of the astronomical twilight before sunrise. The relative weight of the constant streetlight emissions follows the opposite trend. The regulated subset of streetlights seem to have a negligible contribution to the zenithal brightness at the measurement site and their individual curves are not displayed in this figure. 
Figure 6. Normalized time courses, $T_{k}(t)$, of the zenithal night sky brightness (SQM radiance) and the urban emissions from vehicles and residential buildings in the town of Arteixo, estimated from the measurements carried out in the night of 24 to 25 February 2017.

Figure 7. 4th degree polynomial fit of the residential and vehicle light signals shown in Figure 6, which were used as $T_{k}(t)$ for these sources.

Figure 8. The measured SQM zenithal sky brightness of Arteixo, in absolute luminance units $\left(\mathrm{mcd} / \mathrm{m}^{2}\right)$, and its constant streetlights, regulated streetlights, vehicles, residential, and industrial components estimated by Equation (4). SQM(reconstr) is the reconstructed zenithal luminance obtained by adding up the estimated contribution of these components according to Equation (1).

Figure 9. Relative weight of the components of the zenithal sky brightness troughout the night, based on the measurements carried out in Arteixo. As it was observed in A Coruña, the contributions of vehicle and residential lights diminish steadily from the end of the astronomical twilight after sunset until about 04:00 h (CEST), increasing from then on until the beginning of the astronomical morning twilight. The relative weight of the constant streetlight emissions follows the opposite trend. 